BRAVZILIAN JOURNAL

OF MEDICAL AND BIOLOGICAL RESFARCH

www.bjournal.com.br
ISSN 0100-879X

Volume 43 (3) 182-267 March 2011

BIOMEDICAL SCIENCES

AND

CLINICAL INVESTIGATION

Braz J Med Biol Res, March 2011, Volume 44(3) 186-192

doi: 10.1590/S0100-879X2011007500002

Construction of a recombinant adenovirus co-expressing truncated human prostate-specific membrane antigen and mouse 4-1BBL genes and its effect on dendritic cells

Weng Xiaodong, Kuang Youlin, Liu Xiuheng, Chen Zhiyuan, Zhu Hengcheng, Chen Hui, Jiang Botao and Shen Hao

The Brazilian Journal of Medical and Biological Research is partially financed by

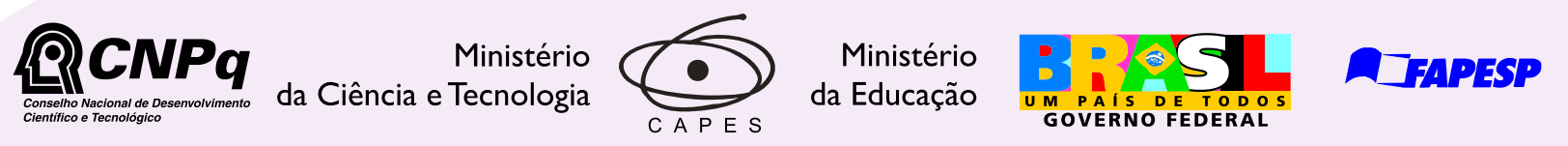

Institutional Sponsors
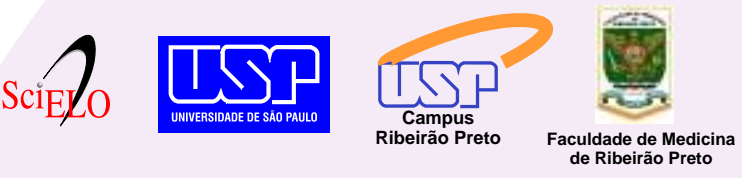

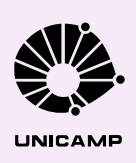

$\oplus$ SHIMADZU

GE Healthcare
Hotsite of proteomics metabolomics developped by:

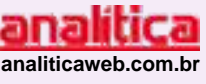

Thermo SCIENTIFIC 


\title{
Construction of a recombinant adenovirus co-expressing truncated human prostate-specific membrane antigen and mouse 4-1BBL genes and its effect on dendritic cells
}

\author{
Weng Xiaodong*, Kuang Youlin*, Liu Xiuheng, Chen Zhiyuan, Zhu Hengcheng, \\ Chen Hui, Jiang Botao and Shen Hao \\ Department of Urology, Renmin Hospital of Wuhan University, Wuhan University, Wuhan, China
}

\begin{abstract}
Our aim was to construct a recombinant adenovirus co-expressing truncated human prostate-specific membrane antigen (tPSMA) and mouse 4-1BBL genes and to determine its effect on dendritic cells (DCs) generated from bone marrow suspensions harvested from C57BL/6 mice for which the effect of 4-1BBL on DCs is not clear, especially during DCs processing tumor-associated antigen. Replication deficient adenovirus AdMax ${ }^{\mathrm{TM}}$ Expression System was used to construct recombinant adenovirus Ad-tPSMA-internal ribosome entry site-mouse 4-1BBL (Ad-tPSMA-IRES-m4-1BBL) and Ad-enhanced green fluorescent protein. Day 7 proliferating DC aggregates generated from C57BL/6 mice were collected as immature DCs and further mature DCs were obtained by lipopolysaccharide activated immature DCs. After DCs were exposed to the recombinant adenovirus with 250 multiplicity of infection, the expression of IPSMA and m4-1BBL proteins were detected by Western blot, and the apoptosis and phenotype of DCs were analyzed by flow cytometry. Cytokines (IL-6 and IL-12) in the supernatant were detected by enzyme-linked immunosorbent assay (ELISA). Proliferation of T cells was detected by allogeneic mixed lymphocyte reactions. The tPSMA and m4-1BBL proteins were expressed correctly. The apoptosis rate of DCs transfected with Ad-tPSMAIRES-m4-1BBL was $14.6 \%$ lower than that of control DCs. The expression of co-stimulatory molecules [CD80 (81.6 $\pm 5.4 \%)$ and CD86 (80.13 $\pm 2.81 \%)]$ up-regulated in Ad-tPSMA-IRES-m4-1BBL-pulsed DCs, and the level of IL-6 (3960.2 $\pm 50.54 \mathrm{pg} /$ $\mathrm{mL})$ and IL-12 (249.57 $\pm 12.51 \mathrm{pg} / \mathrm{mL})$ production in Ad-tPSMA-IRES-m4-1BBL-transduced DCs were significantly higher $(\mathrm{P}<$ $0.05)$ than those in control DCs. Ad-tPSMA-IRES-m4-1BBL induced higher T-cell proliferation $\left(\mathrm{OD}_{450}=0.614 \pm 0.018\right)$, indicating that this recombinant adenovirus can effectively enhance the activity of DCs.
\end{abstract}

Key words: Prostate cancer; PSMA; Co-stimulatory molecule; 4-1BBL; Adenovirus; Dendritic cells

\section{Introduction}

Prostate-specific membrane antigen (PSMA) is an overexpressed membrane-bound cell surface protein on prostate cancer cells (1) and a well-defined tumor-associated antigen (TAA) (2). Because of these properties, PSMA has been proposed as an ideal target for a variety of therapeutic approaches in prostate cancer including the delivery of immunoconjugates, immunotherapy, and prodrugs (3-5). Dendritic cells (DCs) are highly efficient and specialized antigen-presenting cells (APC) that play a central role in immunity (6). DC-based vaccines have been shown to be well suited to induce significant immune responses against prostate cancer $(7,8)$. Of course, DC signaling for a tumorspecific T-cell immune response involves a number of costimulatory molecules, which may amplify, sustain, and drive diversity in the ensuing immune response (9). 4-1BBL is one of well-characterized co-stimulatory molecules expressed on APC (10). 4-1BBL with its receptor 4-1BB expressed on T cells has profound effects on $T$ cells, including activation of both $\mathrm{CD}^{+}$and $\mathrm{CD} 8^{+} \mathrm{T}$ cells, enhanced expansion $(11,12)$, increased long-term survival $(13,14)$, and anti-apoptosis of

Correspondence: Liu Xiuheng, Department of Urology, Renmin Hospital of Wuhan University, 238 Jiefang Road, Wuhan 430060,

China. Fax: +86-27-8804-2292. E-mail: Ixh670@163.com

*These authors contributed equally to this study.

Received August 24, 2010. Accepted December 17, 2010. Available online January 14, 2011. Published March 7, 2011. 
activation-induced $\mathrm{CD}^{+} \mathrm{T}$ cells (15). However, the effect of $4-1 B B L$ on DCs is not clear. In the present study, we constructed a recombinant adenovirus (Ad) co-expressing a TAA and mouse 4 (m4)-1BBL genes and determined the effects of $4-1 B B L$ on DCs during TAA processing.

\section{Material and Methods}

\section{Material}

Female 6- to 8-week-old C57BL/6 $(\mathrm{H}-2 \mathrm{~Kb})$ mice were obtained from Shanghai Slac Laboratory Animal Co., Ltd. (China). Animals were maintained at the Central Animal Facility of Wuhan University according to standard guidelines and experiments were conducted according to the guidelines of the China Council for Animal Care. HEK 293, a human embryonic kidney 293 cell line, was kindly provided by the Ministry of Education Key Laboratory of Virology (Wuhan, China). All cells were cultured in RPMI-1640 medium with $10 \%$ heat-inactivated FCS, 2 mM L-glutamine, $100 \mathrm{U} / \mathrm{mL}$ penicillin, and $100 \mu \mathrm{g} / \mathrm{mL}$ streptomycin at $37^{\circ} \mathrm{C}$ in a humidified atmosphere containing $5 \% \mathrm{CO}_{2}$.

\section{Construction of recombinant co-expression adenovirus vector containing tPSMA and m4-1BBL genes}

The Ad vectors used in the present study were derived from serotype 5. The AdMax ${ }^{\mathrm{TM}}$ Expression System (Microbix Biosystems, Inc., Canada) was used. The Ad-enhanced green fluorescent protein (Ad-eGFP) vector carries the cDNA of eGFP under the transcriptional control of the immediate early promoter of cytomegalovirus (CMV).

Ad-tPSMA-IRES-m4-1BBL carries the cDNA of truncated human PSMA (tPSMA), internal ribosome entry site (IRES) and m4-1BBL. The vector was constructed by inserting sequentially the cDNAs for tPSMA, IRES and m41BBL into the backbone pBHGlox_E1,3Cre. The cDNA of m4-1BBL was taken from the pcDNA3-m4-1BBL plasmid provided by Dr. Tania Watts (Department of Immunology, University of Toronto, Canada), and inserted into the plasmid pDC316-VP3-IRES-sEndo at the Notl/Nhel-restriction sites, creating pDC316-VP3-IRES-m4-1BBL. The cDNA for the extracellular domain of human tPSMA was taken from the pCR3. $1^{\circledR}$-Uni-hPSMA plasmid, a kind gift from Dr. Xiangzhong Yu (Department of Biological Sciences, Clemson University, USA), and inserted into the plasmid pDC316-IRES-eGFP-lacZ at the Agel/Sacl-restriction sites, creating pDC316-tPSMA-IRES-eGFP. The tPSMA fragment was then digested from pDC316-tPSMA-IRES-eGFP with a restriction enzyme and subcloned into pDC316-VP3IRES-m4-1BBL, resulting in pDC316-tPSMA-IRES-m4$1 \mathrm{BBL}$. The entire expression cassette was inserted into the pBHGlox_E1,3Cre backbone by homologous recombination in HEK 293 cells. All adenoviruses were propagated in HEK 293 cells and purified with $\mathrm{CsCl}$ gradients by ultracentrifugation.

\section{DC generation and infection}

Mouse DCs were generated from bone marrow suspensions harvested from 6- to 8-week-old C57BL/6 mice as described in a previous publication (16), with slight modifications. Briefly, bone marrow cells were harvested from femurs and tibiae, depleted of red blood cells, and washed twice in phosphate-buffered saline (PBS). Cells were resuspended in DC medium consisting of RPMI 1640 supplemented with $10 \%$ heat-inactivated FCS (Gibco, USA), $10 \mathrm{ng} / \mathrm{mL}$ GMCSF (R\&D Systems, USA), $10 \mathrm{ng} / \mathrm{mL}$ IL-4 (R\&D Systems), and $50 \mathrm{mM}$ 2-mercaptoethanol, $100 \mathrm{IU} / \mathrm{mL}$ penicillin, and $100 \mu \mathrm{g} / \mathrm{mL}$ streptomycin, and cultured $\left(37^{\circ} \mathrm{C}, 5 \% \mathrm{CO}_{2}\right)$ on 6 -well plates at $1 \times 10^{6}$ cells. $3 \mathrm{~mL}^{-1}$.well-1. On days 3 and 5 of culture, floating cells were gently removed, and fresh $\mathrm{mGM}-\mathrm{CSF} / \mathrm{mlL}-4$-containing medium was added. On day 7, non-adherent cells and loosely adherent proliferating DC aggregates were collected as immature DCs (iDCs) or were activated with $1 \mu \mathrm{g} / \mathrm{mL}$ lipopolysaccharide (LPS, Sigma, USA) for $24 \mathrm{~h}$ to obtain mature DCs.

For Ad transduction, day 7 iDCs were washed and plated onto a 24-well plate containing $200 \mu \mathrm{L}$ serum-free medium supplemented with $10 \mathrm{ng} / \mathrm{mL}$ GM-CSF and $10 \mathrm{ng} /$ $\mathrm{mL} \mathrm{IL-4}$. Virus was added to the wells at 250 multiplicity of infection (MOI) and transduction was allowed to proceed for $2 \mathrm{~h}$ at $37^{\circ} \mathrm{C}$ in $5 \% \mathrm{CO}_{2}$. Complete medium was then added and cells were cultured for an additional $48 \mathrm{~h}$. The transduction efficiency of DCs was checked on the basis of eGFP expression by flow cytometry.

\section{Western blot analysis}

For the detection of tPSMA and m4-1BBL protein expression, $2 \times 10^{6}$ iDCs were infected with recombinant adenoviruses (250 MOI). Two days later, cells were lysed and subjected to SDS-PAGE. Then, protein was transferred to a nitrocellulose membrane (Amersham, USA) and the transferred membrane was probed with polyclonal goat anti-PSMA antibody or goat polyclonal anti-4-1BBL antibody (USA), followed by a horseradish peroxidase-conjugated anti-goat IgG secondary antibody (USA). Antibodies on the membrane were visualized by chemiluminescence (Pierce, USA). Western blot for $\beta$-actin was used as an internal sample.

\section{Surface marker analysis of DCs}

Forty-eight hours after Ad transduction and another 24-h activation with LPS, DCs were stained for $30 \mathrm{~min}$ on ice with FITC- or PE-labeled monoclonal antibodies specific for CD11c, MHC II, CD80, CD86 (BD Pharmingen, Germany). After washing three times in PBS, the cells were analyzed by flow cytometry. Isotype-matched monoclonal antibodies were used as controls.

\section{Determination of cytokine profile by ELISA}

To determine the effect of Ad-tPSMA-IRES-m4-1BBL on the secretion of IL-6 and IL-12 from DCs, supernatants 
were harvested and the concentrations of IL- 6 and IL-12 were measured by ELISA. A mouse IL-6 Quantikine ELISA Kit (R\&D Systems) and a mouse IL-12 Quantikine ELISA Kit (R\&D Systems) were used, respectively. All procedures and conditions were consistent with manufacturer instructions.

\section{Apoptosis analysis by flow cytometry}

For apoptosis analysis, $5 \times 10^{5}$ iDCs were cultured on a 12-well plate containing $500 \mu \mathrm{L}$ complete medium supplemented with $10 \mathrm{ng} / \mathrm{mL}$ GM-CSF and $10 \mathrm{ng} / \mathrm{mL} \mathrm{IL}-4$. Forty-eight hours after Ad transduction, $1 \mu \mathrm{g} / \mathrm{mL}$ of the activating agent LPS and $0.1 \mu \mathrm{g} / \mathrm{mL}$ of the apoptosis inducer bortezomib (Velcade, UK) were added to each well. After an additional 16-h culture, DCs were collected and stained with FITC-conjugated annexin $V$ and propidium iodide according to manufacturer instructions, for apoptosis analysis by flow cytometry (Apoptosis Kit, BD Pharmingen, Germany).

\section{Allogeneic mixed lymphocyte reactions}

Mixed leukocyte reaction (MLR) was performed using three types of LPS-matured DCs (Ad-tPSMA-IRES-m41BBL-transduced DCs, Ad-eGFP-transduced DCs and normal control DCs) as stimulator cells and T lymphocytes as responder cells. Stimulator cells were incubated with 50 $\mathrm{ng} / \mathrm{mL}$ mitomycin $\mathrm{C}$ at $37^{\circ} \mathrm{C}$ for $30 \mathrm{~min}$ and then washed twice with PBS. Nylon wool-purified naive $T$ cells derived from the spleen of allogeneic BALB/c mice were plated onto a 96-well round-bottomed culture plate (Costar, USA) at $4 \times 10^{5}$ cells per well. Stimulators were then added and

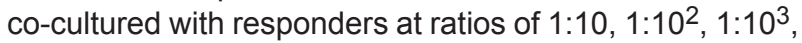
and $1: 10^{4}$ in complete RPMI 1640 medium. DCs and T cells incubated in medium alone served as stimulator control and responder control, respectively. After incubation for 4 days, $10 \mu \mathrm{L}$ of the Cell Counting Kit-8 (Dojindo, Japan) solution was added to each well containing $100 \mu \mathrm{L}$ medium for 4 h. Absorbance was measured at $450 \mathrm{~nm}$ on an automatic ELISA reader (TRITURUS, Spain). All determinations were carried out in triplicate and repeated three times.

\section{Statistical analysis}

Data are reported as means \pm SD and were analyzed by ANOVA or the Student $t$-test, with the level of significance set at $P<0.05$. The SPSS13.0 software was used for statistical analysis.

\section{Results}

\section{Construction of recombinant adenoviruses and transduction of DCs with adenovirus}

A replication-deficient adenovirus vector carrying the human tPSMAgene and m4-1BBL gene (Ad-tPSMA-IRESm4-1BBL) or Ad-eGFP was constructed. The tPSMA and eGFP genes were driven by the CMV promoter, and the translation of the m4-1BBL gene was initiated from an IRES (Figure 1A). DCs were transduced with the adenovirus at $250 \mathrm{MOI}$ to analyze the transduction efficiency. About $65 \%$ of DCs were positive for eGFP expression by flow cytometry (Figure 1B). $250 \mathrm{MOI}$ was considered to be optimal for gene transduction because cell viability was 93\% (Figure 1B).

\section{Assay of tPSMA and m4-1BBL protein}

The expression of $\mathrm{PPSMA}$ and $\mathrm{m} 4-1 \mathrm{BBL}$ in iDCs transduced with Ad-tPSMA-IRES-m4-1BBL was confirmed by Western blotting. The tPSMA and $\mathrm{m4}-1 \mathrm{BBL}$ protein were detected in Ad-tPSMA-IRES-m4-1BBL-transduced DCs but not in Ad-eGFP-transduced DCs or normal control DCs (Figure 2).

\section{Analysis of DC phenotype}

iDCs were infected with Ad-tPSMA-IRES-m4-1BBL, AdeGFP for $48 \mathrm{~h}$, or not infected, and activated with LPS for
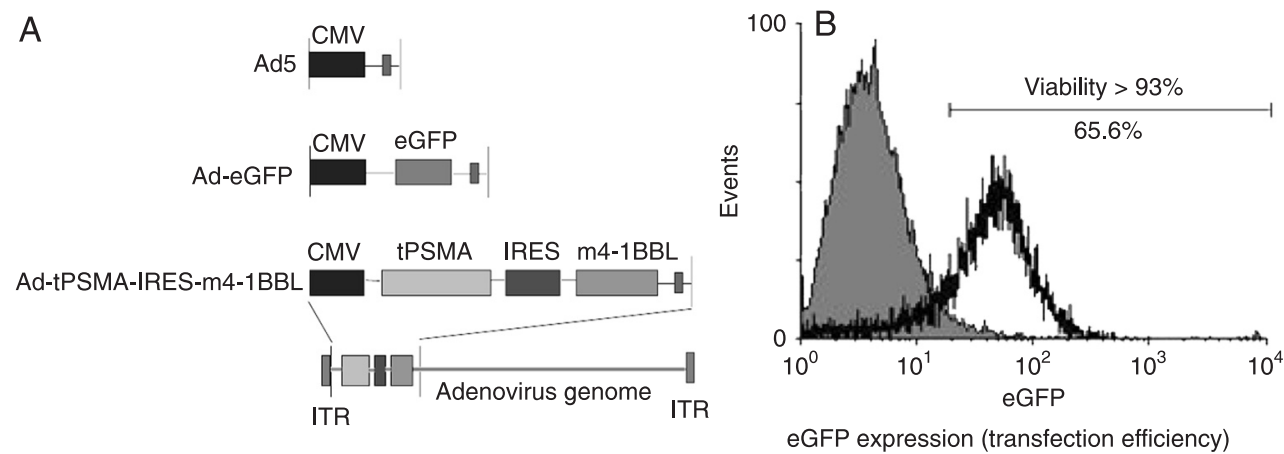

Figure 1. Construction of recombinant adenoviruses (Ad) and transduction of dendritic cells (DCs) with adenovirus. A, Schematic overview of Ad5, Ad-eGFP recombinant adenovirus, and Ad-tPSMA-IRESm4-1BBL recombinant adenovirus. $B$, Transduction efficiency in DCs. eGFP expression was evaluated by flow cytometry $48 \mathrm{~h}$ after gene transduction (65.6\% of eGFP-positive cells). The percentage of viable cells was evaluated by Trypan blue staining. CMV = cytomegalovirus; ITR = inverted terminal repeat. For other abbreviations, see legend to Table 1. 
Table 1. Flow cytometry analysis of dendritic cell (DC) surface markers (\%).

\begin{tabular}{lcccc}
\hline Group & \multicolumn{1}{c}{ CD11c } & MHC II & \multicolumn{1}{c}{ CD80 } & \multicolumn{1}{c}{ CD86 } \\
\hline DCs & $75.46 \pm 1.05$ & $56.93 \pm 5.63$ & $51.84 \pm 4.1$ & $54.63 \pm 2.4$ \\
DCs/Ad-eGFP & $75.80 \pm 1.3$ & $64.33 \pm 3.63$ & $60.50 \pm 6.89$ & $64.20 \pm 0.87$ \\
DCs/Ad-tPSMA-IRES-m4-1BBL & $76.30 \pm 2.5$ & $87.06 \pm 4.75^{*}$ & $81.60 \pm 5.4^{*}$ & $80.13 \pm 2.81^{*}$ \\
\hline
\end{tabular}

The results shown are representative of the percentage of DCs staining positive for each marker and are reported as means \pm SD of three separate experiments. Adenovirus truncated human prostate-specific membrane antigen-internal ribosome entry site-mouse 4-1BBL (AdtPSMA-IRES-m4-1BBL)-infected DCs expressed higher levels of surface markers than Ad-enhanced green fluorescent protein (Ad-eGFP)-transfected DCs and untransfected DCs except for CD11c for which all cells were the same $\left({ }^{*} P<0.05, t\right.$-test).

another $24 \mathrm{~h}$. Then, the expression of CD11c, MHC II, CD80, and CD86 was analyzed by flow cytometry. AdtPSMA-IRES-m4-1BBL-infected DCs enhanced the rate of expression of MHC II, CD80, and CD86 (Table 1).

\section{Cytokine analysis}

The culture supernatants of DCs transfected with AdtPSMA-IRES-m4-1BBL, Ad-eGFP, or uninfected DCs were collected for the analysis of IL- 6 and IL-12 production by ELISA. The concentration of IL- 6 and IL-12 in Ad-tPSMAIRES-m4-1BBL-transfected DC culture supernatants was higher than in DCs transfected with Ad-eGFP and untreated DCs (Figure 3).

\section{Analysis of DC apoptosis}

iDCs transfected with Ad-tPSMA-IRES-m4-1BBL, AdeGFP, or uninfected DCs were treated with the activating agent LPS and the apoptosis inducer bortezomib. After 16 $\mathrm{h}$ of culture, DCs were collected and stained with FITCconjugated annexin $\mathrm{V}$ and propidium iodide for detecting apoptosis. The rate of apoptosis of Ad-tPSMA-IRES-m41BBL-transduced DCs was lower than that of Ad-transduced and uninfected DCs (Figure 4).

\section{Proliferation}

DCs are potent stimulators of primary MLRs and are able to induce the proliferation of allogeneic T lymphocytes in vitro. We compared the abilities of our DC populations to stimulate primary MLRs among allogeneic $T$ lymphocytes. The data demonstrated that DCs transduced with Ad-tPSMA-IRES-m4-1BBL induced stronger allogeneic T-cell proliferative responses in vitro than untreated DCs and DCs transduced with Ad-eGFP (Figure 5).

\section{Discussion}

DCs are among the most potent APCs for induction of antitumor immune responses currently known and have been recognized as potentially important tools for cancer vaccine strategies (17). Bone marrow-derived DCs have

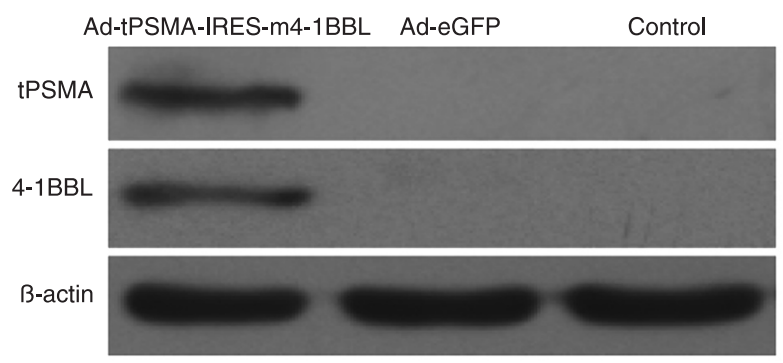

Figure 2. Detection of tPSMA protein and $\mathrm{m4}-1 \mathrm{BBL}$ protein by Western blot. Total cell lysates were prepared and the presence of tPSMA protein and $\mathrm{m4}-1 \mathrm{BBL}$ protein was detected using the anti-PSMA polyclonal antibody and anti-4-1BBL polyclonal antibody, respectively. A tPSMA-specific band and a 4-1BBL-specific band were detected in dendritic cells (DCs) transfected with AdtPSMA-IRES-m4-1BBL but not in DCs transfected with Ad-eGFP or untreated DCs. For abbreviations, see legend to Table 1.

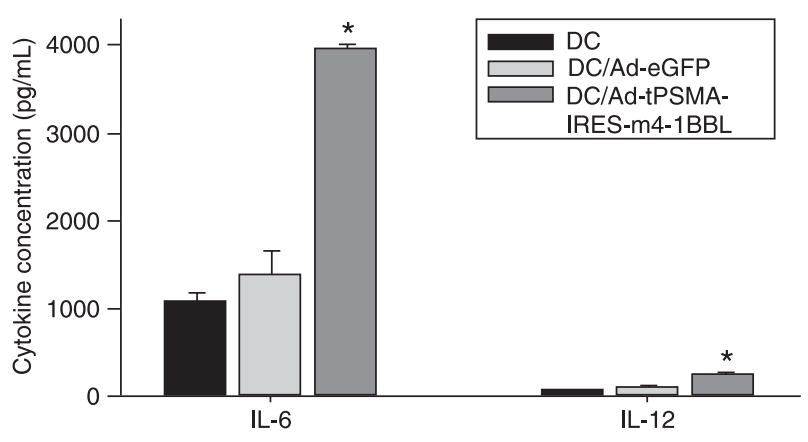

Figure 3. Cytokine (IL-6/IL-12) production by dendritic cells (DCs). The culture supernatants of DCs transfected with AdtPSMA-IRES-m4-1BBL, Ad-eGFP, or not transfected were collected for the analysis of IL-6 and IL-12 production by ELISA. Data are reported as means $\pm S D$. Similar results were obtained from three independent experiments. ${ }^{*} \mathrm{P}<0.05$ compared to $\mathrm{DCs}$ transfected with Ad-eGFP and non-transfected DC ( $t$-test). For abbreviations, see legend to Table 1. 


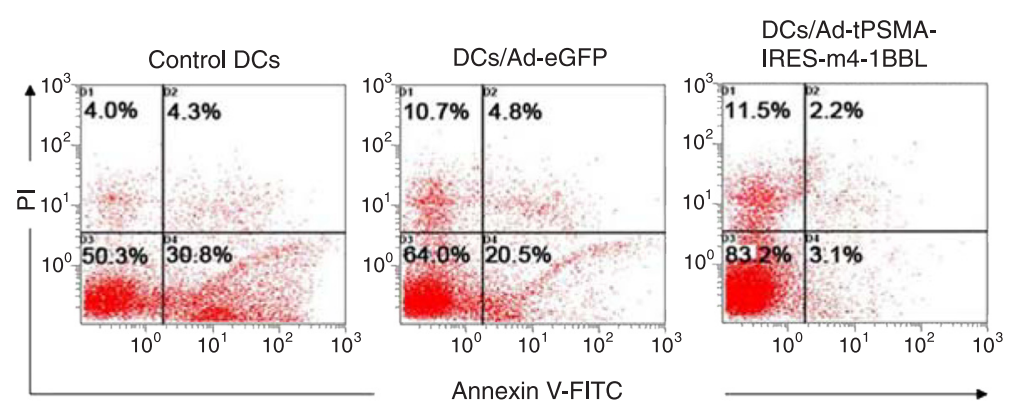

Figure 4. Analysis of dendritic cell (DC) apoptosis. Immature DCs transfected with Ad-tPSMA-IRES-m4-1BBL, Ad5, or not transfected were treated with the activating agent lipopolysaccharde and the apoptosis inducer bortezomib. After 16 $\mathrm{h}$ of culture, DCs were collected and stained with FITC-conjugated annexin V and propidium iodide (PI) for apoptosis analysis by flow cytometry. The results are representative of three independent experiments. For abbreviations, see legend to Table 1.

been successfully employed in vitro both in animal models and in clinical trials $(18,19)$. Moreover, genetic material can be introduced into DCs with varying levels of efficacy, using techniques such as electroporation, lipid-mediated transfection, calcium phosphate precipitation, and virally mediated gene transfer (20-22). Recombinant adenovirus is the most efficient vector for gene transfer to DCs due to its two unique features. First, adenovirus performs a transgene delivery to DCs of higher magnitude than other available systems (23). In addition, adenovirus infection alone, without the addition of a therapeutic transgene, causes a high degree of DC maturation in terms of phenotype and function (24). Our results showed that the expression of eGFP in transfected DCs reached a high level of $65 \%$ (Figure 1B), which indicated efficient gene transduction.

PSMA is a well-defined prostate-restricted TAA whose expression is significantly elevated in carcinoma of the prostate, especially in advanced stages. It has been shown that a PSMA-encoding adenovirus-transfected DC vaccine induced a specific and strong immune response against prostate cancer cells (25). 4-1BBL, with its receptor 4-1BB, forms a pair of co-stimulatory molecules with profound effects on $T$ cells, including enhancement of T-cell expansion, and increased T-cell effector function $(16,26,27)$. 4-1BBL gene-modified DCs could enhance effector and memory cytotoxic T-lymphocyte responses $(28,29)$. Recently, immature DCs have been reported to constitutively express 4-1BB (30), raising the possibility of DC-DC reciprocal and/or autocrine stimulation via the 4-1BB/4-1BBL pathway. Thus, 4-1BBL may be involved in $\mathrm{DC}$ activation in a $\mathrm{T}$ cell-independent, as well as a $\mathrm{T}$ cell-dependent fashion (30).

In the present study, we tried to analyze the immunogenicity of DCs transduced with recombinant adenovirus carring a TAA gene and m4-1BBL gene. Our results demonstrated the higher expression of surface

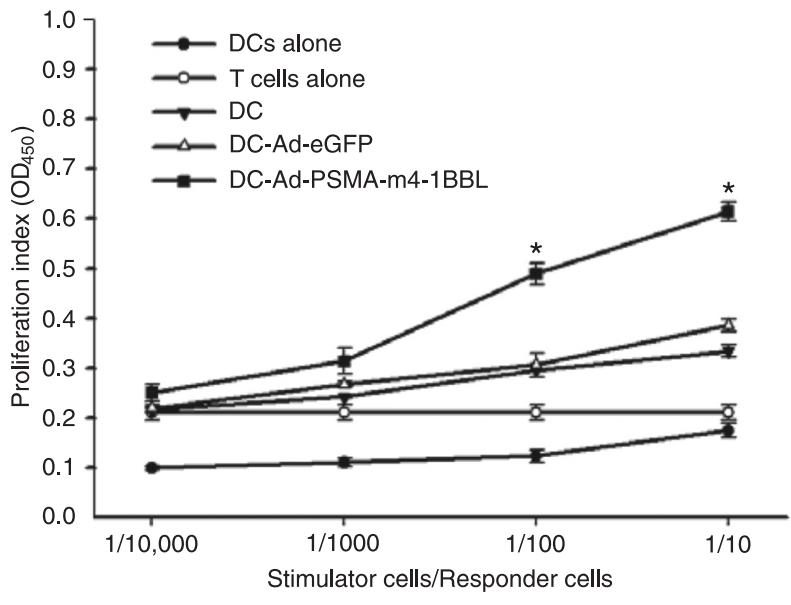

Figure 5. Mixed lymphocyte reaction. T lymphocytes were stimulated with Ad-tPSMA-IRES-m4-1BBL-infected dendritic cells (DCs), Ad-eGFP-transfected DCs, and non-transfected DCs. DCs transfected with Ad-tPSMA-IRES-m4-1BBL for $48 \mathrm{~h}$ and matured with lipopolysaccharide for another $24 \mathrm{~h}$ were collected and co-cultured with T cells for $96 \mathrm{~h}$. Specific cytotoxic T lymphocytes were detected by the cholecystokin-8 assay. Data are reported as means $\pm S D$ of three replicates. The data indicate that Ad-tPSMA-IRES-m4-1BBL-infected DCs were potent lymphocyte stimulators compared to Ad-eGFP-transfected DCs and non-transfected DCs ( ${ }^{*} \mathrm{P}<0.05$ compared to Ad-eGFP-transfected DCs and non-transfected DCs; $t$-test). For abbreviations, see legend to Table 1.

markers (MHC II, CD80, CD86) on DCs transduced with Ad-tPSMA-IRES-m4-1BBL than on DCs transfected with Ad-eGFP and non-transfected DCs (Table 1). This higher expression of surface molecules may enhance antigen presentation to $T$ cells by DCs. Ad-tPSMA-IRES-m41BBL-transduced DCs significantly increased cytokine (IL-6, IL-12) production (Figure 3) and T-cell proliferation. 
We also used the apoptosis inducer bortezomib $(0.1 \mu \mathrm{g} /$ $\mathrm{mL}$ ) to treat Ad-transfected DCs and the results indicated that Ad-tPSMA-IRES-m4-1BBL-transduced DCs showed higher anti-apoptosis ability (Figure 4). The present results demonstrate that recombinant adenovirus co-expressing truncated human prostate-specific membrane antigen and m4-1BBL gene-modified DCs enhanced immunogenicity, which may induce a specific and strong immune response against prostate cancer cells. Further studies are needed to address this possibility.

\section{References}

1. Tiffany CW, Lapidus RG, Merion A, Calvin DC, Slusher BS. Characterization of the enzymatic activity of PSM: comparison with brain NAALADase. Prostate 1999; 39: 28-35.

2. Horoszewicz JS, Leong SS, Kawinski E, Karr JP, Rosenthal $\mathrm{H}$, Chu TM, et al. LNCaP model of human prostatic carcinoma. Cancer Res 1983; 43: 1809-1818.

3. Bühler P, Wetterauer D, Gierschner D, Wetterauer U, Beile UE, Wolf $P$. Influence of structural variations on biological activity of anti-PSMA scFv and immunotoxins targeting prostate cancer. Anticancer Res 2010; 30: 3373-3379.

4. Bühler P, Wolf $P$, Gierschner D, Schader I, Katzenwadel A, Schultze-Seemann W, et al. A bispecific diabody directed against prostate-specific membrane antigen and CD3 induces T-cell mediated lysis of prostate cancer cells. Cancer Immunol Immunother 2008; 57: 43-52.

5. Kularatne SA, Venkatesh C, Santhapuram HK, Wang K, Vaitilingam B, Henne WA, et al.Synthesis and biological analysis of prostate-specific membrane antigen-targeted anticancer prodrugs. J Med Chem 2010; 53: 7767-7777.

6. Nencioni A, Grunebach F, Schmidt SM, Muller MR, Boy D, Patrone $F$, et al. The use of dendritic cells in cancer immunotherapy. Crit Rev Oncol Hematol 2008; 65: 191-199.

7. Sonpavde G, Spencer DM, Slawin KM. Vaccine therapy for prostate cancer. Urol Oncol 2007; 25: 451-459.

8. Kim S, Lee JB, Lee GK, Chang J. Vaccination with recombinant adenoviruses and dendritic cells expressing prostatespecific antigens is effective in eliciting CTL and suppresses tumor growth in the experimental prostate cancer. Prostate 2009; 69: 938-948.

9. O'Neill DW. Dendritic cells and T cells in immunotherapy. $J$ Drugs Dermatol 2010; 9: 1383-1392.

10. Schwarz H. Biological activities of reverse signal transduction through CD137 ligand. J Leukoc Biol 2005; 77: 281286.

11. Laderach D, Movassagh M, Johnson A, Mittler RS, Galy A. 4-1BB co-stimulation enhances human CD8(+) T cell priming by augmenting the proliferation and survival of effector CD8(+) T cells. Int Immunol 2002; 14: 1155-1167.

12. Lu ZY, Condomines M, Tarte K, Nadal L, Delteil MC, Rossi JF, et al. B7-1 and 4-1BB ligand expression on a myeloma cell line makes it possible to expand autologous tumorspecific cytotoxic T cells in vitro. Exp Hematol 2007; 35: 443-453.

13. Lee HW, Park SJ, Choi BK, Kim HH, Nam KO, Kwon BS. 4-1BB promotes the survival of $\mathrm{CD} 8+\mathrm{T}$ lymphocytes by

\section{Acknowledgments}

Special thanks are due to Dr. Xiangzhong Yu (Department of Biological Sciences, Clemson University, USA) for providing the pCR3. $1^{\circledR}$-Uni-hPSMA vector, to Dr. Tania Watts (Department of Immunology, University of Toronto, Canada) for providing pcDNA3-m4-1BBL, to Dr. Jianguo Wu (Ministry of Education Key Laboratory of Virology, Wuhan University) and to the Vector Gene Technology Company Ltd. Research supported by the National Natural Science Foundation of China (Grants \#30672107, \#30901494, and \#30901552).

increasing expression of Bcl-xL and Bfl-1. J Immunol 2002; 169: 4882-4888.

14. Croft M. Co-stimulatory members of the TNFR family: keys to effective T-cell immunity? Nat Rev Immunol 2003; 3: 609620.

15. Kudo-Saito C, Hodge JW, Kwak H, Kim-Schulze S, Schlom J, Kaufman HL. 4-1BB ligand enhances tumor-specific immunity of poxvirus vaccines. Vaccine 2006; 24: 4975-4986.

16. Cannons JL, Lau P, Ghumman B, DeBenedette MA, Yagita $\mathrm{H}$, Okumura $\mathrm{K}$, et al. 4-1BB ligand induces cell division, sustains survival, and enhances effector function of CD4 and CD8 T cells with similar efficacy. J Immunol 2001; 167: 1313-1324

17. Rolinski J, Hus I. Dendritic-cell tumor vaccines. Transplant Proc 2010; 42: 3306-3308.

18. Nagaraj S, Pisarev V, Kinarsky L, Sherman S, Muro-Cacho C, Altieri DC, et al. Dendritic cell-based full-length survivin vaccine in treatment of experimental tumors. $J$ Immunother 2007; 30: 169-179.

19. Kraemer M, Hauser S, Schmidt-Wolf IG. Long-term survival of patients with metastatic renal cell carcinoma treated with pulsed dendritic cells. Anticancer Res 2010; 30: 20812086.

20. Tian S, Liu Z, Donahue C, Noh HS, Falo LD Jr, You Z. Transcriptional IL-15-directed in vivo DC targeting DNA vaccine. Gene Ther 2009; 16: 1260-1270.

21. Oh ST, Kim CH, Park MY, Won EH, Sohn HJ, Cho HI, et al. Dendritic cells transduced with recombinant adenoviruses induce more efficient anti-tumor immunity than dendritic cells pulsed with peptide. Vaccine 2006; 24: 2860-2868.

22. Melhem NM, Gleason SM, Liu XD, Barratt-Boyes SM. Highlevel antigen expression and sustained antigen presentation in dendritic cells nucleofected with wild-type viral mRNA but not DNA. Clin Vaccine Immunol 2008; 15: 1337-1344.

23. Di Nicola M, Siena S, Bregni M, Longoni P, Magni M, Milanesi $\mathrm{M}$, et al. Gene transfer into human dendritic antigenpresenting cells by vaccinia virus and adenovirus vectors. Cancer Gene Ther 1998; 5: 350-356.

24. Hirschowitz EA, Weaver JD, Hidalgo GE, Doherty DE. Murine dendritic cells infected with adenovirus vectors show signs of activation. Gene Ther 2000; 7: 1112-1120.

25. Kraaij R, van Rijswijk AL, Oomen MH, Haisma HJ, Bangma $\mathrm{CH}$. Prostate specific membrane antigen (PSMA) is a tissuespecific target for adenoviral transduction of prostate cancer in vitro. Prostate 2005; 62: 253-259. 
26. Sluijter BJ, van den Hout MF, Stam AG, Lougheed SM, Suhoski MM, van den Eertwegh AJ, et al. 4-1BB-mediated expansion affords superior detection of in vivo primed effector memory CD8(+) T cells from melanoma sentinel lymph nodes. Clin Immunol 2010; 137: 221-233.

27. Habib-Agahi M, Jaberipour M, Searle PF. 4-1BBL costimulation retrieves CD28 expression in activated T cells. Cell Immunol 2009; 256: 39-46.

28. Wiethe C, Dittmar K, Doan T, Lindenmaier W, Tindle R. Provision of $4-1 B B$ ligand enhances effector and memory CTL responses generated by immunization with dendritic cells expressing a human tumor-associated antigen. $J$ Immunol 2003; 170: 2912-2922.

29. Sharma RK, Schabowsky RH, Srivastava AK, Elpek KG, Madireddi S, Zhao H, et al. 4-1BB ligand as an effective multifunctional immunomodulator and antigen delivery vehicle for the development of therapeutic cancer vaccines. Cancer Res 2010; 70: 3945-3954.

30. Futagawa T, Akiba H, Kodama T, Takeda K, Hosoda Y, Yagita $\mathrm{H}$, et al. Expression and function of 4-1BB and 4-1BB ligand on murine dendritic cells. Int Immunol 2002; 14: 275-286. 